\title{
THE CONTROL SYSTEM OF THE ACTIVE MAIN REFLECTOR FOR FAST
}

\author{
YUHAI QIU and LICHUN ZHU \\ National Astronomical Observatories, Chinese Academy of Sciences, Beijing 100012, China
}

\begin{abstract}
The main reflector of FAST consists of about 1800 elementary units. Each unit has three adjustable supports to fix its position, and its position is adjusted by means of mechanical actuators. According to the radio source position at any given time, all the actuators are continuously adjusted to form a proper paraboloid in real time during the course of the observation. The basic requirements of such a control system are discussed. A fieldbus control system based on LonWorks technology is suggested to control all 1800 actuators. The main advantages of this distributed control system are its reliability, flexibility, efficiency and economics.
\end{abstract}

Keywords: Spherical Radio telescope, LonWorks control system

\section{Introduction}

FAST (Five hundred meter Aperture Spherical radio Telescope) will be the world's largest radio telescope; it is proposed to be built in the unique karst area in the southwest of China (Peng et al., 2000). The main anticipated features of FAST are as follows:

- Main spherical reflector:

Curvature radius: 300m; Physical diameter: 500m and opening angle: 120 degree.

- Available aperture: $300 \mathrm{~m}$

- Sky coverage: $>40$ degrees

- Full polarization and multi-beam capability

- Working frequency: from $200 \mathrm{MHz}$ to $5 \mathrm{GHz}$

- The sensitivity: 9 times better than GBT

The novel design for this giant spherical radio telescope which is proposed by Qiu (Qiu, 1998), may be described as 'Arecibo type with active main mirror'. The part of the spherical mirror illuminated by the feed is continuously adjusted to form a proper paraboloid of revolution ( $300 \mathrm{~m}$ aperture) in real time, during the course of the observation. To fit this parabola, it is necessary to divide the main mirror into about 1800 smaller units. Each unit is a small part of the spherical surface and is a hexagon with sides about $7.5 \mathrm{~m}$ long. Each one has three adjustable supports to fix its position and link it with the two adjacent units by means of a special connector. Altogether there would be about 1800 supports (Figure 1). The support with its 


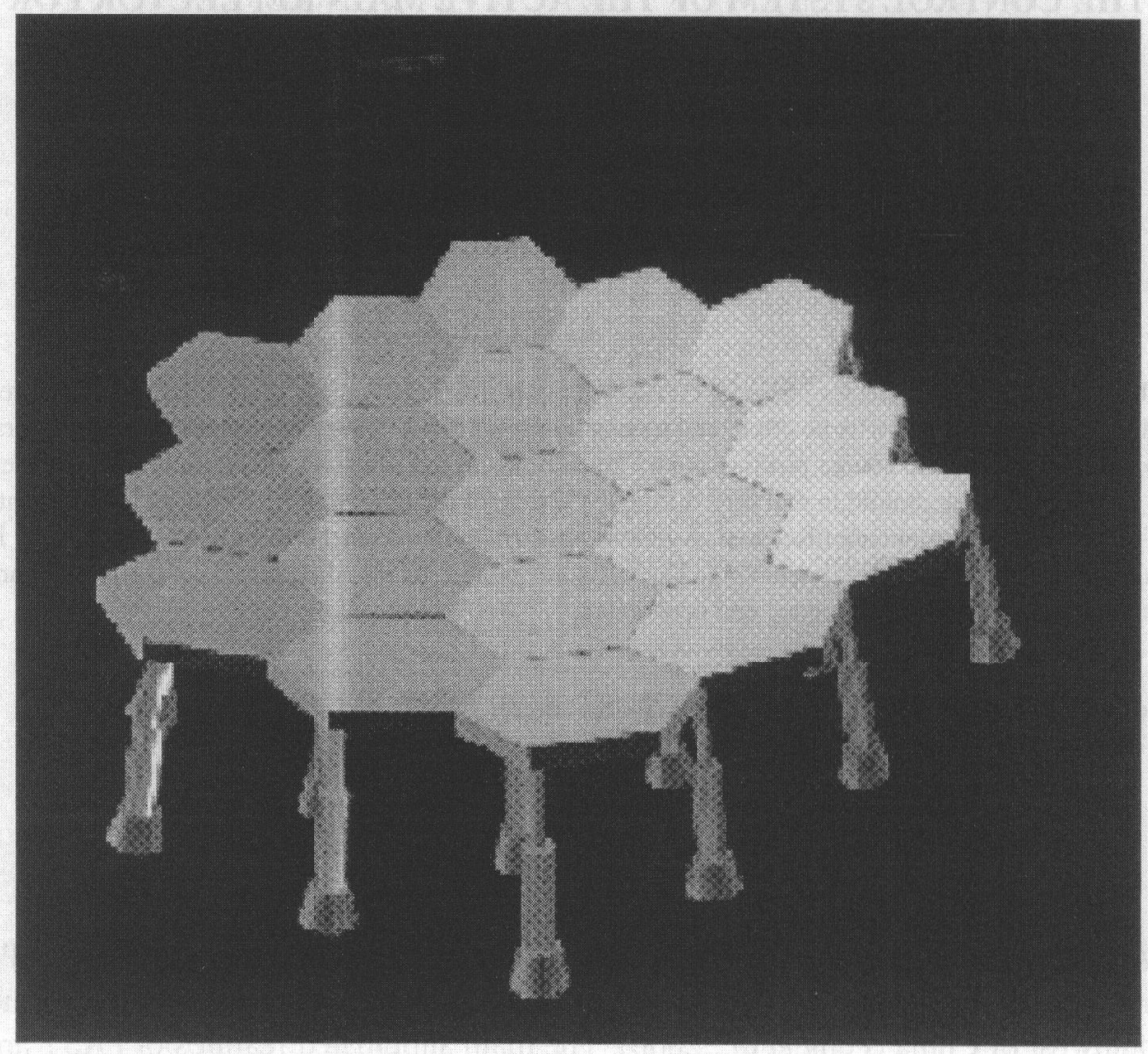

Figure 1. The hexagon units and their supports with actuators.

actuator is directed towards the center of the sphere. The position of each unit is adjustable by means of actuators. The maximum range of height variation for any actuator is $67 \mathrm{~cm}$ and the maximum rate of variation is $4.4 \mathrm{~cm} / \mathrm{min}$ for tracking the object. With this design an important task for us is to develop an economical, reliable, flexible and efficient control system which can adjust all 1800 actuators in real time.

\section{Basic Requirements of the Control System}

According to the source position at any time, the heights of all actuators are computed and then the actuators are continuously adjusted to form an instantaneous paraboloid during tracking of the source. Following Qiu's analysis (Qiu, 1998) the control may be effected in steps, say $1 \mathrm{~mm}$ at a time, this gives a quantization error of $0.5 \mathrm{~mm}$ which may be neglected when working at $5 \mathrm{GHz}$. The step rate of $1 \mathrm{~Hz}$ is enough to match the requirement of maximum rate of support variation when 
tracking the source; while slewing to a new target can be achieved in less than 12 minutes. Thus the main requirements of this control system are as follows:

- To determine the height of all actuators during observation in real time

- To check the position of all actuators $(0.1 \mathrm{~mm})$ in real time when required by the central control computer

- To control the necessary actuators to move up/down one step $(1 \mathrm{~mm})$ at the rate of $1 \mathrm{~Hz}$ for fitting the instantaneous paraboloid

- A memory function for zero position (i.e. a spherical figure) for all actuators

- Alarm function when any error occurs

- Remote control via Ethernet.

\section{Main Principles of the Control System}

Some principles for this control system are suggested as follows:

- High reliability, flexibility, efficiency and economics

- The modern industrial automatic technology is applied

- A real distributed control system with intelligent nodes

- EMS compatibility

- Friendly interface

\section{Description of the Control System based on LonWorks Technology}

Considering the discussion above, a control system based on one of the up-todate field bus technologies - LonWorks - is suggested. LonWorks technology developed by Echelon Corporation since 1988 makes possible a new generation of smart control products which working together can form a control network. The LonTalk protocol that is implemented in firmware on the Neuron chip provides the foundation for LonWorks interoperability.

The control system based on the LonWorks technology is shown in Figure 2. The system consists of two levels. The upper level is the master computer and the lower level consists of about 1800 intelligent nodes, connected with the master computer via a network. The master computer may consist of several Industrial Personal Computers. The main tasks of the master computer are:

- To make the observation schedule

- To calculate the heights of all desired actuators when pointing and tracking the radio source in real time

- To send commands to the intelligent nodes to move the actuators

- To check the position of all actuators

- To handle the alarm events from the front end when any error occurs

- To connect with remote computers via Ethernet 


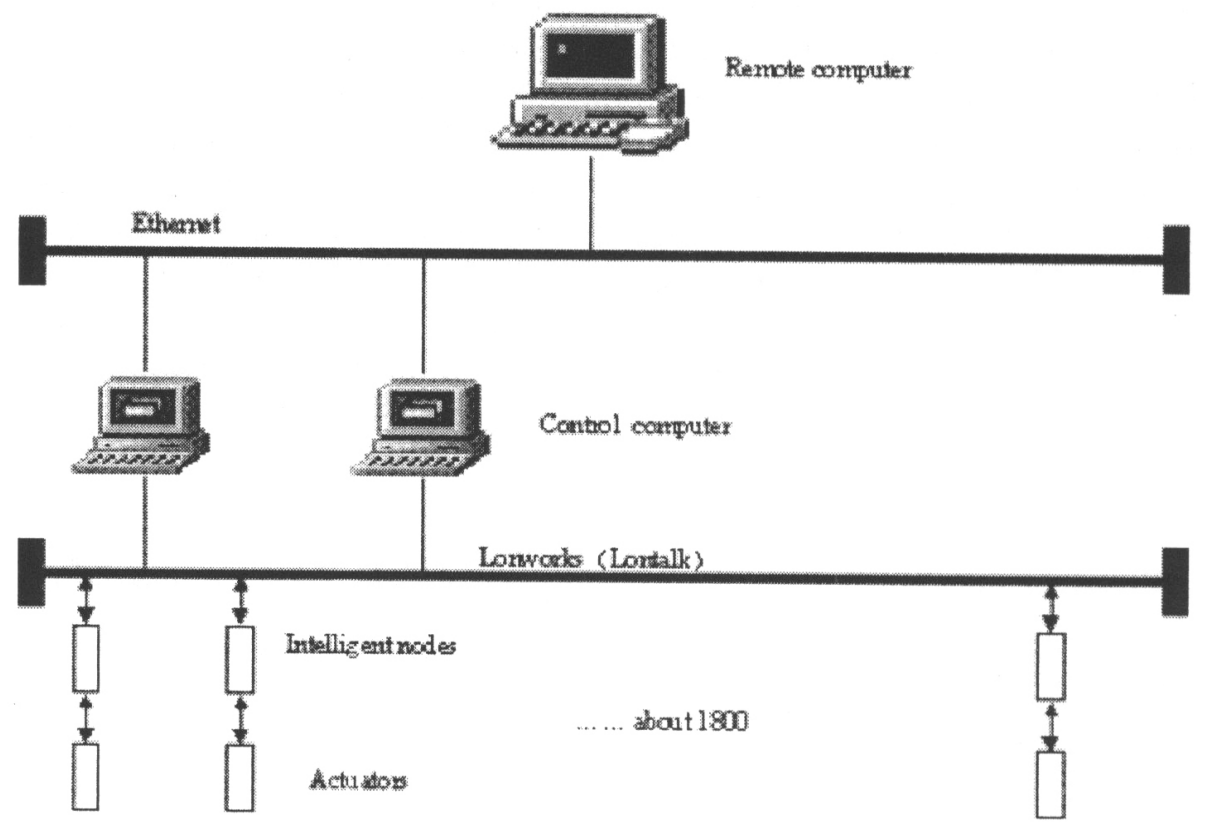

Figure 2. The block diagram of the control system for the active main reflector.

The intelligent node is based on a Neuron chip; it includes a Neuron chip, transceiver, memory and support hardware. The Neuron chip executes the application program and implements the LonTalk protocol. It will fit our requirements. Computer programs that run on Neuron chips are developed using the Neuron C programming language. The main tasks of the intelligent nodes are as following:

- To communicate with the master computer via fibre-optic or twisted-pair cable

- To control the movement of actuators

- To get the position information of all actuators

- To handle the error events

LonWorks has all the advantages of fieldbus technology. Also, LonWorks control systems offer several key competitive advantages over other control systems, with the result that LonWorks control systems are more cost-effective, more easily maintained and expanded, and more versatile in the functions they can perform. LonWorks control networks offer a feature called 'distributed processing' whereby each device in the network can receive, transmit and process network information independently of other devices. This means that devices in a LonWorks control system can make decisions and process information without the need for a PC, programmable logic controller (PLC), or some other form of central host processor. Eliminating the need for a central processor reduces the total cost of LonWorks systems. It also makes the system more reliable since a failed device does not shut down the rest of the control network, as would happen if a PLC or central 
host processor were to fail. Another key advantage of LonWorks control system is that the protocol, or language, used to communicate between devices is an open, published protocol accredited by international standard bodies under standards such as EIA709 and IEEE 1473. In the open world of LonWorks, there are multiple sources of supply and customers have the freedom to choose their sources of supply and maintenance. In order to offer maximum flexibility, LonWorks were designed from the ground up to operate on multiple communications media such as twisted pair, power line, fibre-optic, radio frequency, infrared and coaxial cable. LonWorks control systems also support different means of wide area networking, including IP and permit remote clients to communicate over IP networks for remote operation works.

\section{References}

Peng, B., et al.: 2000, ApSS, in press.

Qiu, Y.: 1998, A novel design for a giant Arecibo-type Spherical radio telescope with an active main reflector, MNRAS 301, 827-830. 